\title{
Index of Plantar Pressure Alters with Prolonged Diabetes Duration
}

Lei Xu $\cdot$ Hui Zeng $\cdot$ Jun Zhao $\cdot$ Jungong Zhao $\cdot$ Jun Yin •

Hua Chen $\cdot$ Yimin Chai $\cdot$ Yuqian Bao $\cdot$ Fang Liu $\cdot$ Weiping Jia

Received: July 31, 2019 / Published online: October 8, 2019

(C) The Author(s) 2019

\section{ABSTRACT}

Introduction: Diabetic foot ulcers develop with deviations in the distribution of plantar pressure. It is difficult to interpret any alteration in plantar pressure under different conditions of type 2 diabetes mellitus (T2DM). The aim of this study was to gain a better insight into the

Enhanced Digital Features To view enhanced digital features for this article go to https://doi.org/10.6084/ m9.figshare.9861017.

L. Xu · H. Zeng · J. Yin · Y. Bao · F. Liu ( $₫) \cdot$ W. Jia Shanghai Key Laboratory of Diabetes, Department of Endocrinology and Metabolism, Shanghai Clinical Medical Center of Diabetes, Shanghai Key Clinical Center of Metabolic Diseases, Shanghai Institute for Diabetes, Multi-disciplinary Collaboration Diabetic Foot Group Shanghai Diabetes Institute, Shanghai Jiao-Tong University Affiliated Sixth People's Hospital, Shanghai, China e-mail: f-liu@sjtu.edu.cn

\section{J. Zhao}

Department of Vascular Surgery, Shanghai JiaoTong University Affiliated Sixth People's Hospital, Shanghai, China

J. Zhao

Department of Interventional Radiology, Shanghai Jiao-Tong University Affiliated Sixth People's Hospital, Shanghai, China

H. Chen · Y. Chai

Department of Orthopedics, Shanghai Clinical Medical Center of Orthopedics, Shanghai Jiao-Tong University Affiliated Sixth People's Hospital,

Shanghai, China variations in plantar pressure with increased duration of diabetes.

Methods: Plantar pressure was examined in 1196 participants with or without T2DM. Subjects with normal glucose tolerance (NGT) and impaired glucose tolerance (IGT) were assigned to control groups, and those with T2DM were divided into five groups according to diabetes duration ( $<2$ years, 2-5 years, 5-10 years, $10-15$ years, and $>15$ years). The clinical characteristics, plantar peak pressure, and pressure-time integral (PTI) were compared among the seven study groups, and factors associated with peak pressure and the PTI were analyzed.

Results: At the hallux, peak pressure exhibited an upward trend in patients with T2DM within 5 years of diabetes duration, followed by a distinct downward slope with further progression of the disease (trend analysis, $p<0.05$ ). An uneven distribution of peak pressure was found at other locations, but this unevenness was ultimately lower than that in the two control groups $(p<0.05)$. No obvious trend was noted for PTI among patients with different diabetes duration; however, those with diabetes for $>10$ years manifested a significantly sharper increase in the PTI at the metatarsus (11.63 $\left.\mathrm{Ns} / \mathrm{cm}^{2}, p<0.05\right)$ and heel $\left(14.12 \mathrm{Ns} / \mathrm{cm}^{2}\right.$, $p<0.05)$ than at the hallux $\left(8.76 \mathrm{Ns} / \mathrm{cm}^{2}\right)$. A fluctuation in the PTI was also detected at the hallux and midfoot of diabetes patients, which was broadly flat when compared with that of 
the two control groups. The stepwise multiple regression analysis revealed that the variation in plantar pressure was independently associated with age, body mass index, and vibration perception threshold (VPT) $(p<0.05)$.

Conclusions: There would appear to be an association between longer diabetes duration and decreased peak pressure for the hallux, suggesting that individuals with diabetes for $>10$ years will have an increased PTI for the metatarsus and heel. The reduced pressure on the hallux is believed to be transferred to the metatarsus. Age, BMI, and VPT are distinct risk factors of abnormal plantar pressure.

Keywords: Diabetes duration; Type 2 diabetes; Plantar pressure; Peak pressure; Pressure-time integral

\section{INTRODUCTION}

Foot problems in diabetes account for more hospital admissions than any of the other longterm complications and are the leading cause of non-traumatic lower extremity amputation [1], resulting in increased morbidity and mortality [2]. Diabetic foot ulcers are caused by a combination of several risk factors [3], with significant contributing roles for elevated mechanical foot pressures and diabetic peripheral neuropathy $[4,5]$. Emerging evidence suggests that elevated plantar pressure is also a significant determinant of foot ulcer recurrence [6]. Consequently, it is imperative that physicians recommend that interventions be routinely taken against abnormal plantar pressure.

There are undoubtably many mechanical and biological factors that could contribute to elevated plantar pressure, such as foot deformity, limited joint mobility, callus, soft tissue thickness, metatarsal length, the configuration of the medial longitudinal arch, movement codes, body mass index (BMI), and obesity [7-9]. Nevertheless, the current interpretation does not sufficiently explain how plantar pressure affects the development of diabetic foot ulcers. A majority of the variance in plantar pressure remains unexplained, despite the significant role of high plantar pressure in ulcer progression. For example, it remains as yet unclear whether diabetes duration plays a role in the occurrence of abnormal plantar pressure or not.

An abundance of studies have incidentally reported the relationship between diabetes duration and plantar pressure distribution in diverse populations. Based on the results of their cross-sectional study of Chinese outpatients, Shen et al. reported that duration of type 2 diabetes mellitus (T2DM) has no significant correlation with the total pressure-time integral (PTI) [7]. Halawa et al. reported similar results in their study of two groups of patients with T2DM with a mean duration of diabetes of 10.4 and 4.9 years (total $n=50$ patients), respectively, and 30 healthy volunteers [10]. On the contrary, one of the global features of a sub-analysis of the DIAFOS trial was the identification of duration of diabetes as a distinct but weak predictor of plantar peak pressure, as compared to local factors, such as the presence of foot deformity [11]. In a study involving north Asian Indian patients with diabetes, the investigators measured plantar pressure distribution using the parameter-power ratio and found that this ratio was higher in patients with a longer duration of diabetes; this index assisted the investigators to distinguish the diabetic neuropathy groups from the control group based on hindfoot and forefoot pressure while standing [12]. To date, little predictive value has been given to the contribution of diabetes duration in terms of plantar pressure distribution in people with diabetes due to the different populations, limited sample sizes, and diversified assessment indexes of the various studies. In addition, to date no data have been found to substantiate the specific effect of diabetes duration on plantar pressure.

Therefore, the aim of our study was to investigate the specific impact of diabetes duration and its exact role in the distribution of abnormal plantar pressure in patients with diabetics. Our hypothesis was that diabetic patients with longer diabetes duration would manifest worse pedobarographic parameters. 


\section{METHODS}

\section{Subjects}

A cross-sectional and observational study was conducted. A total of 1196 subjects (49.7\% male; mean age $62.3 \pm 11.5$ years) with normal glucose tolerance (NGT), impaired glucose tolerance (IGT), and T2DM were recruited from the Shanghai Clinical Medical Center of Diabetes. All individuals were diagnosed with NGT, IGT, or T2DM based on 1999 World Health Organization criteria and American Diabetes Association standards [13]. A history of physiciandiagnosed diabetes was assessed by questionnaire. The prevalence of hypertension (HP), cerebral-cardio vascular diseases (CCVD), smokers, and drinkers were compared between the NGT $(n=37)$, IGT $(n=49)$, and five T2DM groups categorized according diabetes duration (group 1: diabetes duration $<2$ years, $n=238$; group 2: diabetes duration: 2 to $<5$ years, $n=258$; group 3: diabetes duration 5 years to $<10$ years, $n=178$; group 4: diabetes duration 10 to $<15$ years, $n=251$ ); group 5 : diabetes duration $\geq 15$ years, $n=185$ ). Subjects were excluded if they had: (1) secondary lower extremity neuropathy due to lumbar intervertebral disc herniation, lumbar vertebral tumors, cerebral infarction and persistent walking disability, radiotherapy, vitamin B1 deficiency or others; (2) active plantar ulcer or history of diabetic foot ulcers; (3) severe chronic diabetes complications, such as retinopathy-related blindness, end-stage renal disease, and others; (4) acute complications of diabetes; (5) history of stress, such as fever, infection, or trauma during the past 2 weeks; (6) rheumatic, muscular, and articular diseases of the lower limbs, such as rheumatorid arthritis, mono-arthritis, deforming arthrosis, gonarthrosis, myopathies, among others; or (7) abnormalities and deformities of the lower limbs, feet, and spine.

All procedures performed in studies involving human participants were in accordance with the Institutional Review Board and Ethics Committee of Shanghai Sixth people's Hospital affiliated to the Shanghai Jiao Tong University School of Medicine and with the 1964 Helsinki
Declaration and its later amendments or comparable ethical standards. Informed consent was obtained from all individuals included in the study.

\section{Procedures}

Information on sex, age, BMI, waist-to-hip ratio (WHR), diabetes duration, and history of HP and CCVD was collected from all participants. BMI was calculated as body weight $(\mathrm{kg})$ divided by the square of the height $(\mathrm{m})$. Smoking history and alcohol intake were recorded. The analysis of serum biochemical indexes, including alanine aminotransferase (ALT), aspartate aminotransferase (AST), total cholesterol (TC), triglyceride (TG), high-density lipoprotein cholesterol, low-density lipoprotein cholesterol, and serum creatinine was performed by the enzymatic method with an automatic biochemical analyzer (Hitachi Inc., Tokyo, Japan). The creatinine clearance rate (Ccr) was calculated based on the official formula. Levels of fasting plasma glucose (FPG) and 2-h postprandial blood glucose (PPG) were estimated by the glucose oxidase method. Glycosylated hemoglobin (HbA1c) was determined by high-pressure liquid chromatography using the Variant II system (Bio-Rad Inc., Hercules, CA, USA).

A neuropathic assessment of the vibration perception threshold (VPT) was measured by the same technician for all study participants using a neurothesiometer (BioThesiometer; BioMedical Instrument Co., Newbury, OH, USA). The operational approaches were based upon the recommendations of the International Working Group on the Diabetic Foot of the International Diabetes Federation. The test was performed three times per person, and the mean voltage was calculated as the VPT result of this lateral. The higher value of VPT in either limb was selected for further analysis. The ankle-brachial index (ABI), which is the ratio of ankle systolic pressure to arm systolic pressure, was detected according to the standard protocols recommended by the International Diabetes Federation. The lower value of $A B I$ in either limb was selected for further analysis. 
Plantar pressure was monitored using a $50-\mathrm{cm}$ footscan ${ }^{\circledR}$ pressure plate (RSscan International, Olen, Belgium; $250 \mathrm{~Hz} ; 4$ sensors/ $\mathrm{cm}^{2}$ ). The $50-\mathrm{cm}$ footscan pressure measurement plate together with a 1-m external dimension plate is incorporated into the floor to provide a 'complete platform' that is $2.5 \mathrm{~m}$ in length; this design ensures that the second step is taken into data collection each time. The subject walks barefoot at a comfortable walking pace over the footscan ${ }^{\circledR}$ plate, steps onto the platform with their second foot strike, and continues to walk over and past the plate for approximately $1 \mathrm{~m}$. The software automatically divides the plantar into ten masked zones: hallux; toes 2-5 (pressures in toes $2-5$ of all the participants could not be detected, thus, this part was deleted from our analysis); first to fifth metatarsals (M1, M2, M3, M4, and M5); midfoot (MF); medial heel (MH); and lateral heel (LH) (Fig. 1). All parameters of the gait cycle are registered and can be analyzed in great detail using footscan software supplied by RSscan

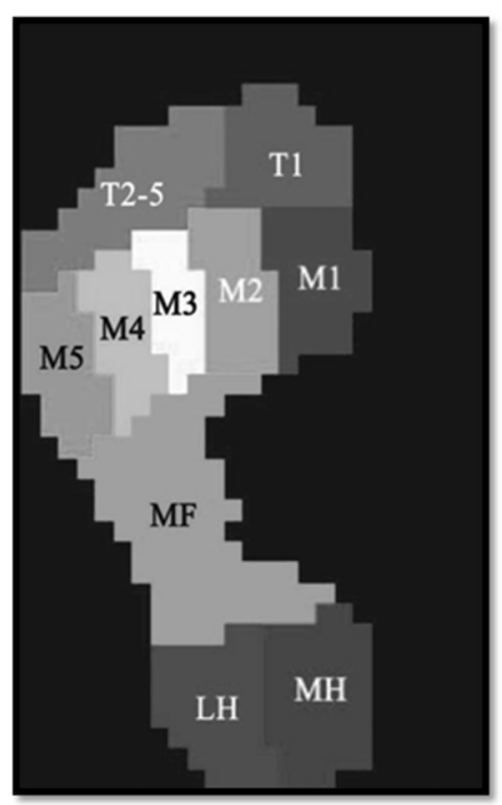

Fig. 1 Schematic drawing of the zones of the foot analyzed in this study. The ten subregions were hallux, toes 2-5 (T2-5), first metatarsal $(M 1)$, second metatarsal (M2), third metatarsal (M3), fourth metatarsal (M4), fifth metatarsal $(M 5)$, midfoot $(M F)$, medial heel $(M H)$, and lateral heel $(L H)$
International. The peak pressure and PTI of the nine regions and the entire foot were calculated and analyzed.

\section{Data Analysis}

Categorical variables were expressed as percentages, and continuous variables were given as mean \pm standard deviation values. Comparison of continuous variables among the seven study groups (NGT group, IGT group [used as controls]; 5 T2DM groups) was performed using one-way analysis of variance. Non-parametric testing was accomplished by the Kruskal-Wallis test. Trend analysis was undertaken to describe the alterations in plantar pressure. Relationships between peak pressure or the PTI and diabetes duration were assessed with Pearson correlation analysis. Associations between peak pressure or PTI and other variables were evaluated with stepwise multiple regression analysis. Statistical analyses were performed using SPSS version 24.0 software (IBM Corp., Armonk, NY, USA). $P<0.05$ was considered to be statistically significant.

\section{RESULTS}

\section{Clinical Characteristics}

Descriptive characteristics for the seven study groups are listed in Table 1. As shown, there were no intergroup differences for gender, BMI, and WHR $(p>0.05)$. Intragroup differences were present for age. The mean age of subjects in the five DMT2 groups increased with increased duration of diabetes (57.18 [group 1] vs. 59.37 [group 2] vs. 61.97 [group 3] vs. 65.06 [group 4] vs. 69.72 years [group 5]; $p<0.01$ ), while the mean ages of subjects with NGT (61.45 years) and IGT (63.31 years) were comparatively older. There were significant differences in the percentages of CCVD and HP and values of the VPT and ABI among the patients in the five T2DM groups with different diabetes duration (all $p<0.05$ ). The incidences of CCVD and HP among T2DM patients showed an upward trend from group 1 to group 5. With 


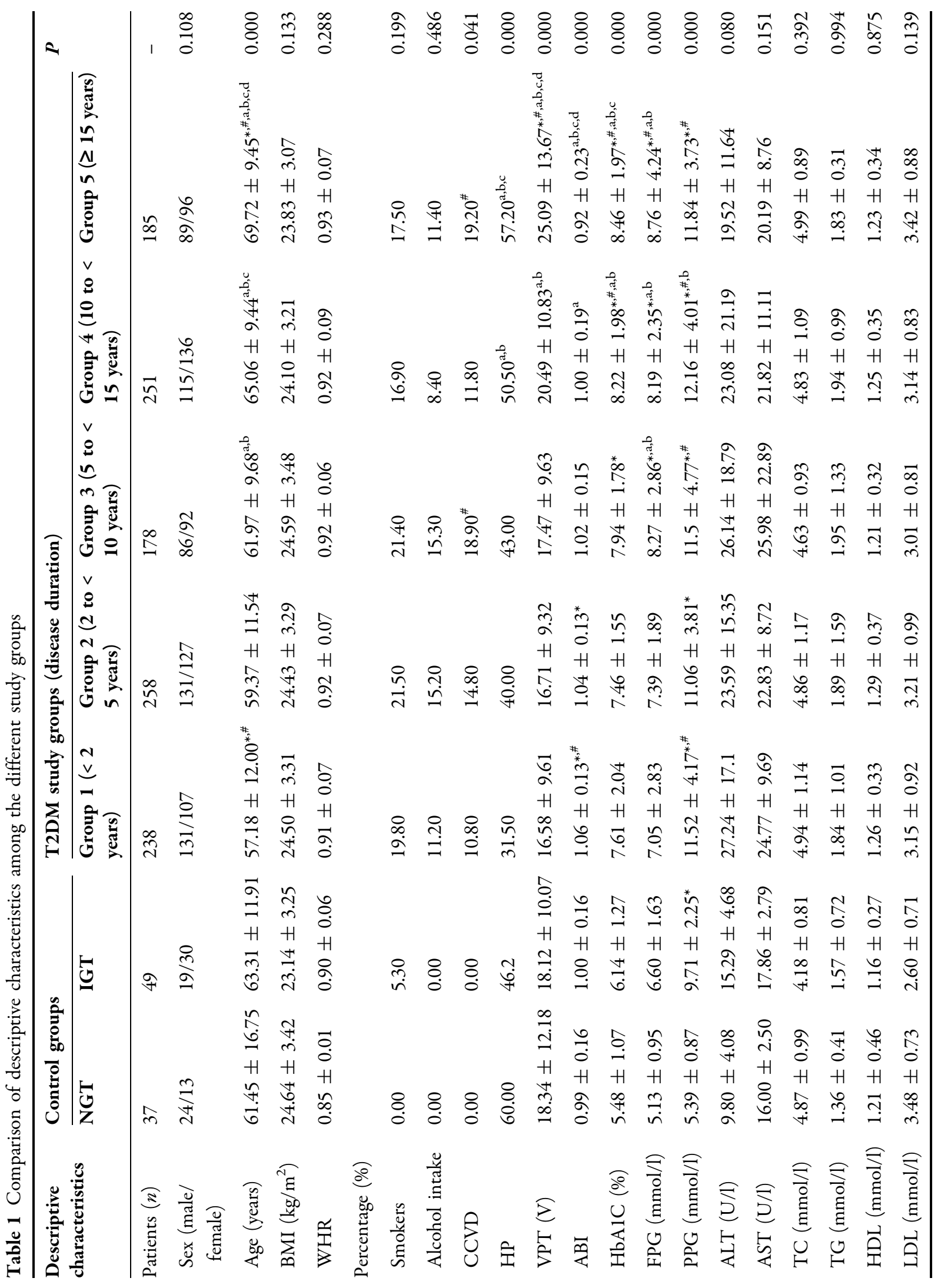




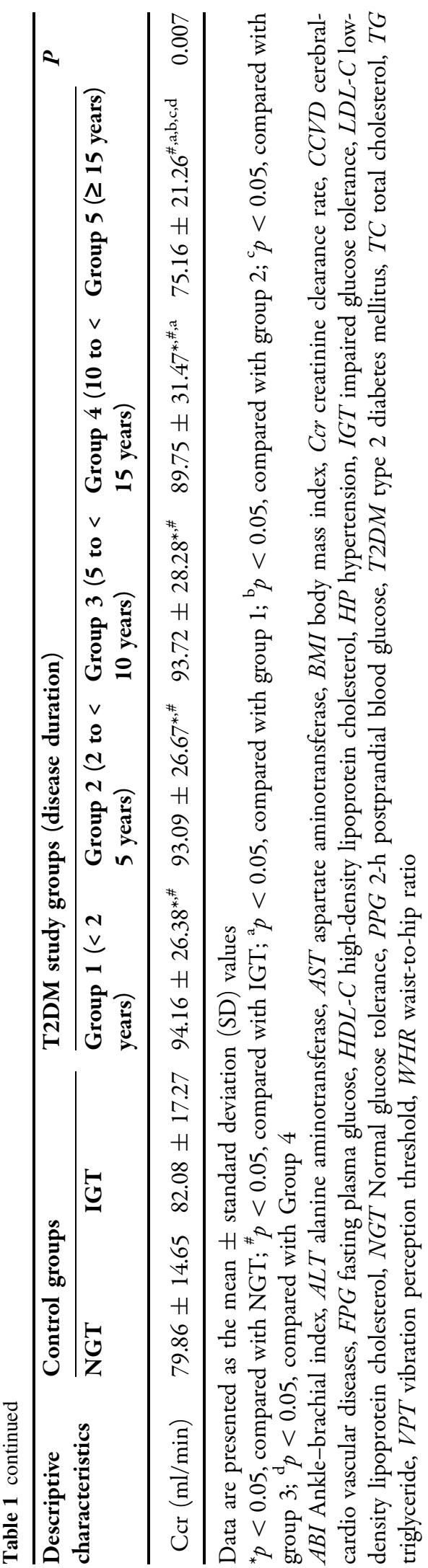

increasing diabetes duration, the VPT in all T2DM groups increased significantly $(p<0.01)$, and the ABI fell significantly $(p<0.01)$. Glycemic control became worse with increasing duration of diabetes based on the gradual rise in HbA1c, FPG, and PPG levels $(p<0.01)$. No statistically significant differences among these groups were found for TC, TD, ALT, and AST $(p>0.05)$. Patients in the five T2DM groups displayed a significant reduction in the Ccr $(p<0.01)$. In general, as the T2DM patients got older, there was a higher prevalence of complications; the biochemical indexes became worse with increasing duration of diabetes. There were relatively higher incidences of CCVD and HP in the NGT and IGT groups, a slightly higher VPT, and a lower ABI, indicating poor conditions due to a relatively advanced age.

\section{Plantar Peak Pressure and Pressure-Time Integral Distribution}

Data analysis was conducted for nine plantar subregions and the entire foot (Figs. 2, 3) for peak pressure and the PTI. No significant difference was found between the left and right foot for all of the measured parameters $(p>0.05)$. Peak pressure and the PTI in both the non-diabetes and diabetes groups were significantly higher for the M2, M3, MH, and $\mathrm{LH}$ subregions of the foot than for the other subregions $(p<0.05)$. As shown in Figs. 2 and 3, almost $57.4 \%$ of the total peak pressure and $56.3 \%$ of the total PTI were distributed at these four subregions in the five diabetes groups, with approximately $10 \%$ of the entire plantar pressure focused on the hallux. Peak pressure or the PTI of the MF each constituted a mere $2-3 \%$ of the total plantar pressure, and both were unevenly distributed $(p>0.05)$.

As shown in Fig. 2, 10.05, 10.32, 10.47, $11.10,10.45,9.90$, and $9.60 \%$ of the total peak pressure in each study group was distributed on the hallux $(51.88,49.70,51.39,55.79,51.00$, 49.14 , and $43.68 \mathrm{~N} / \mathrm{cm}^{2}$, respectively). In the diabetes patients, peak pressure exhibited an upward trend at the hallux within 5 years of diabetes duration, followed by a clear downward slope with further progression of the 

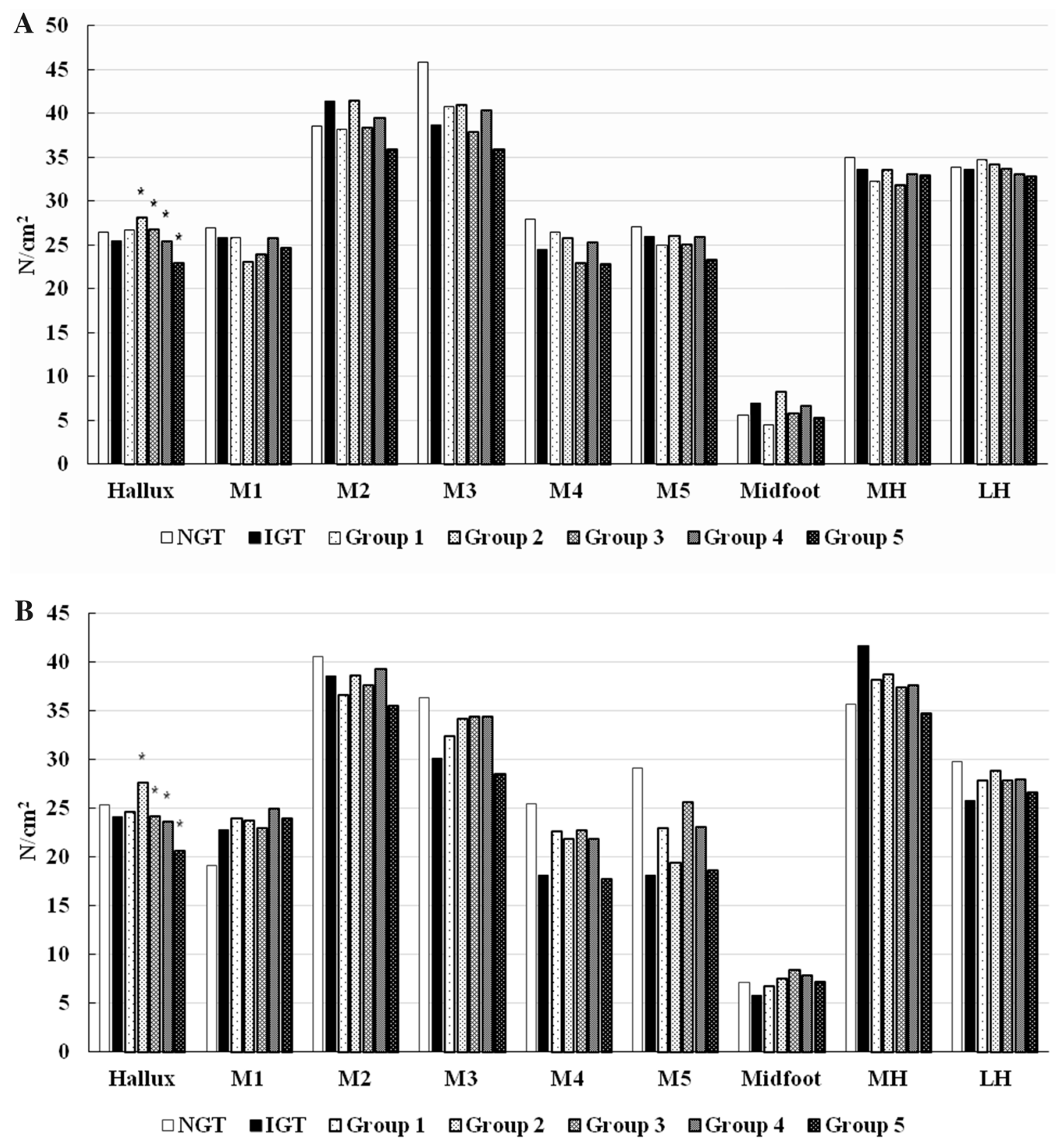

Fig. 2 Distribution of plantar peak pressure among the different study groups. a left foot, $\mathbf{b}$ right foot. Significance: ${ }^{*} p<0.05$ for trend analysis. Approximately 10, 60, 3 , and $27 \%$ of the plantar peak pressure was located at the hallux, metatarsus, $\mathrm{MH}$ and $\mathrm{MH}$, respectively. $N G T$

disease (trend analysis for T2DM groups 2 to 5 , $p<0.05)$. The percentage of peak pressure for all individuals at the metatarsus that was distributed uniformly was around 60\%, and no regular variation was detected. Peak pressure at
Normal glucose tolerance group, IGT impaired glucose tolerance group, Groups 1, 2, 3, 4, 5 subjects with type 2 diabetes mellitus for varying durations $(<2,2$ to $<5,5$ to $<10,10$ to $<15$, and $\geq 15$ years, respectively)

the heel accounted for about $27 \%$ of total peak pressure, sharing a similar distribution pattern as that at the hallux, but the trend analysis yielded no significant results $(p>0.05)$. Overall, peak pressure displayed a final decline with 

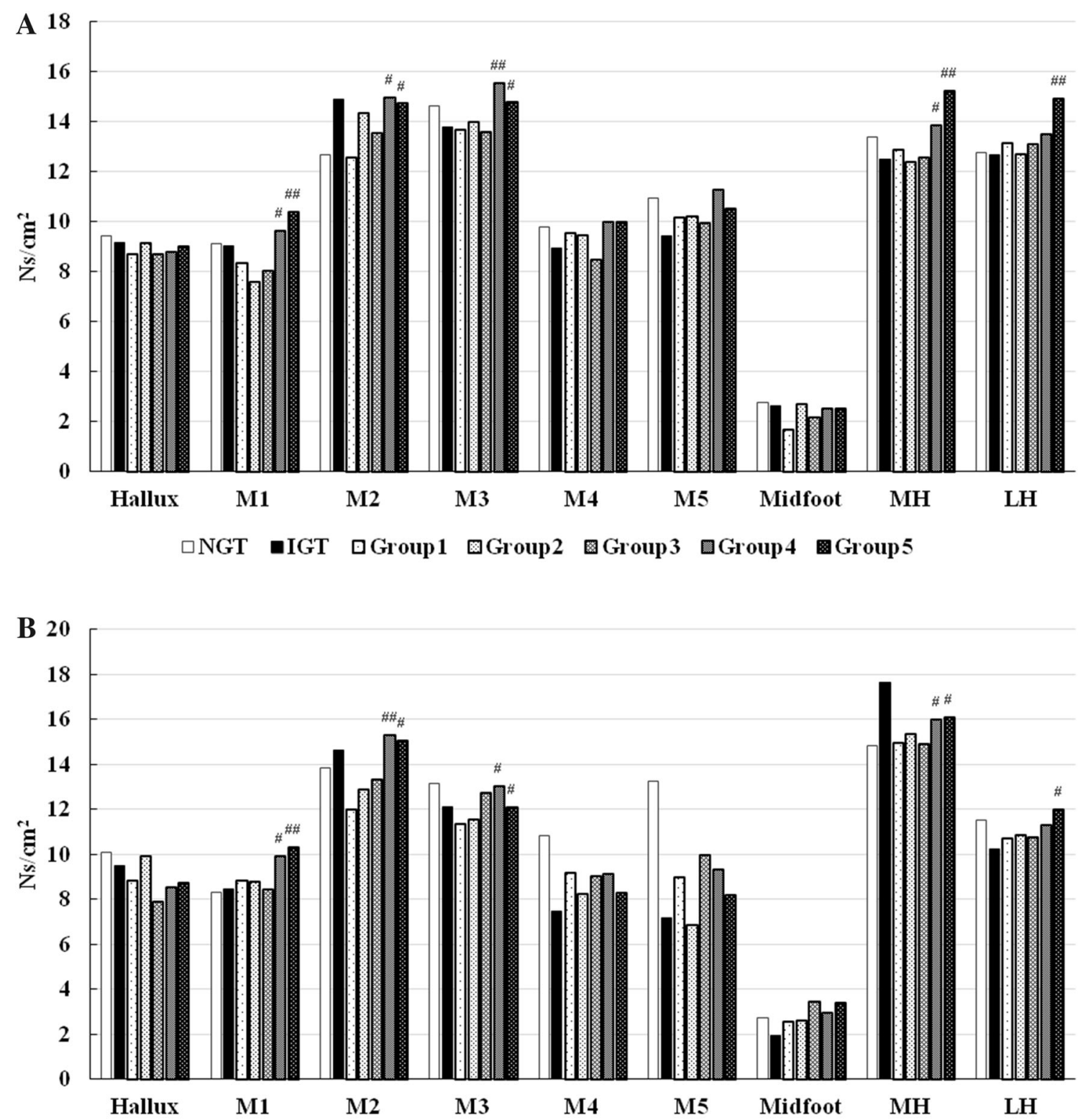

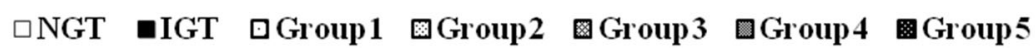

Fig. 3 Distribution of the pressure-time integral of plantar pressure among the different study groups. a left foot, $\mathbf{b}$ right foot. Significance: ${ }^{\#} p<0.05,{ }^{\#} p<0.01$ vs. groups $1-3$. Approximately $9.5,59,3$, and $28.5 \%$ of the

increasing duration of T2DM, predominantly at the hallux ( $p<0.05)$; there were no significant differences in the other plantar regions, but ultimately peak pressure across all diabetes plantar peak pressure was located at the hallux, metatarsus, midfoot, and heel, respectively. See captions to Figs. 1 and 2 for abbreviations

groups was lower than that in the controls $(p<0.05)$.

The distribution of the PTI is summarized in Fig. 3. In each study group, the proportions of hallux accounting for the entire plantar PTI was 


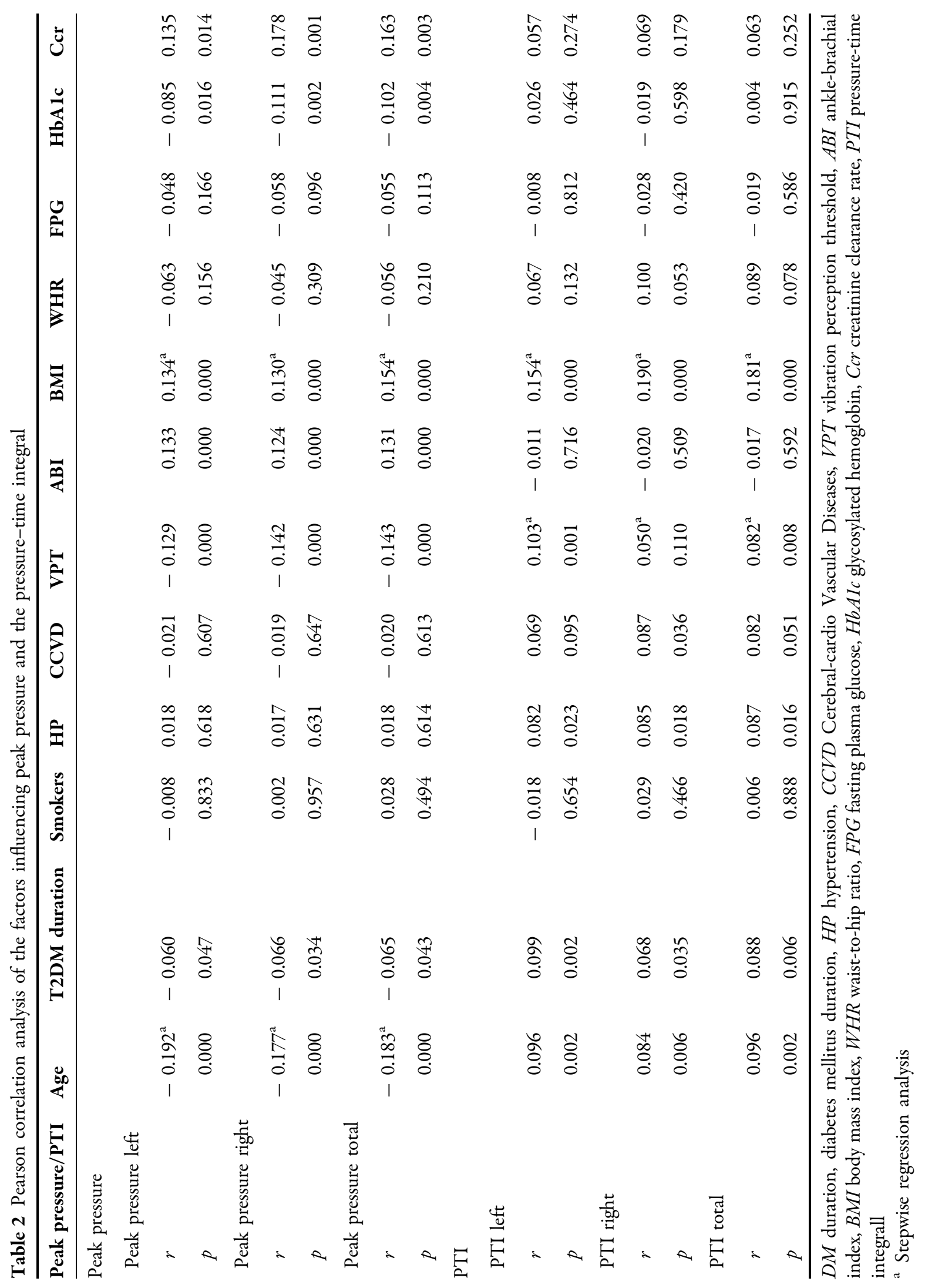


$10.06,10.25,9.83,10.62,9.18,8.84$, and 9.03, respectively $(19.53,18.69,17.53,19.08,16.61$, 17.31 , and $17.74 \mathrm{Ns} / \mathrm{cm}^{2}$, respectively), illustrating that there was no significant diversity $(p>0.05)$. No obvious trend was noted in terms of PTI values among the diabetes subgroups. However, there was a significantly sharp increase in all the analyzed PTIs of the metatarsus and heel for patients with diabetes for $>10$ years (groups 4 and 5; $p<0.05$ ), especially in the M1-3 regions. A fluctuation in the PTI of the hallux and MF was detected, but this was broadly flat when compared with the PTI of the control groups $(p>0.05)$.

\section{Factors Associated with Peak Pressure and the PTI}

The Pearson correlation analysis revealed a significant but low relationship between total peak pressure $(r=-0.065, p=0.043)$ or total PTI $(r=0.088, p=0.006)$ and diabetes duration. The association between plantar pressure and other clinical characteristics among the patients with different diabetes duration is shown in Table 2. No correlation was detected in the nondiabetes subjects due to the relatively small number of controls.

The related characteristics differed among the different T2DM groups. Peak pressure was related to BMI, WHR, ABI, VPT, HbA1c, and FPG (Table 2, all $p<0.05$ ). Stepwise multiple regression analysis also indicated that BMI was able to explain $20.5,21.7$, and $34.1 \%$ of the variance for peak pressure in the hallux, metatarsus, and heel, respectively $(p<0.01)$. A significant correlation between the barefoot PTI and age, BMI, WHR, ABI, VPT, HbA1c, FPG, and Ccr was found (Table 2, all $p<0.05$ ). The parameters of BMI and VPT were able to explain 23.4 and $12.8 \%$, respectively, of the variance for PTI in the metatarsus ( $p<0.05)$, while BMI and age explained 28.5 and $16.2 \%$, respectively of the variance for PTI in the heel $(p<0.01)$.

\section{DISCUSSION}

The results of this study demonstrate an upward trend in peak pressure on the hallux in diabetes patients within 5 years of disease onset, followed by a distinct downward slope with continued duration of the disease. The peak appeared in patients with a duration of T2DM of 2-5 years (group 2 ) due to the early increase in the incidence of mild diabetic neuropathy, which would induce abnormal plantar pressure; peak pressure then decreased gradually with further increase in diabetes duration. We attribute the distribution pattern of peak pressure in our study population of diabetics to the reduced cutaneous sensitivity that results from moderate or severe diabetic neuropathy and arterial disease, two conditions that develop along the course of diabetes, perhaps in combination with callus formation and other known or unknown factors. These results are in agreement with those of another study which reported that a progressive degeneration of peripheral nerves and arteries, especially in the lower limbs, could cause sensory and motor deficits that affect the biomechanics of the foot in diabetes, as seen in ankle kinematics, gait kinetics, and plantar pressure distribution analyses [14-16]. However, reduced plantar cutaneous sensation has also been reported either to lead to no changes or not to be a decisive factor in alterations in plantar pressure while walking [17]. Ultimately, the lower peak pressure in the patients in the T2DM groups in our study compared to the controls confirms the impact of reduced plantar cutaneous sensation on changes in plantar pressure distribution.

Data consistently show that the pressure pattern of the diabetic foot refers only to forefoot, because this is the heavily loaded area where neuropathic ulcers commonly develop, especially the metatarsal heads [18]. A number of factors could explain why the forefoot may experience higher stress than the hindfoot, including soft tissue thickness [19], prominent metatarsal heads in the forefoot [20], among others. Consistently, up to $60 \%$ of the total peak pressure in our diabetic patients was located at the metatarsus; yet, there was no obvious trend in peak pressure at the metatarsus with disease progression. We suggest that due to diabetic peripheral nerve damage and vascular disease, pressure on the metatarsus first decreased, as observed on the hallux, but then extra pressure 
was transferred from the relatively insensitive and flexible hallux, which could not bear too great of a gradual load, leading to the fluctuating distribution on the metatarsus. In agreement with this explanation, it has been reported that patients with neurovascular disease with intact sensation are able to sense high pressures and pain and to adjust their weightbearing activities accordingly [21]. Thus, our explorations could explain why considerable discrepancy occurred with regard to previously shifting results, as well as provide a reasonable interpretation of the association between favorite locations of pressure-related ulcers and plantar pressure distribution [22, 23].

Some evidence has been found to suggest that an early increase in plantar pressure would not necessarily lead to an increase in peak pressure but rather to an increase in the PTI [22]. Recent studies suggest that this integral provides an indication of plantar loading behavior [24]. However, our findings showed no significant variation of the PTI at the hallux of T2DM patients with varying duration of disease. Similarly, other analyses have also found no difference in the PTI for the forefoot between diabetes patients with and without foot ulcers [4]. However, a number of studies have reported an extremely higher PTI value in control groups relative to neuropathic groups that allowed the latter to be distinguished $[17,25]$. Moreover, it would appear that approximately 50\% of diabetic patients will develop diabetic peripheral neuropathy within 10-15 years of acquiring the disease [17]. In this context, we observed a distinct increase in the PTI among our diabetes subjects with $>10$ years of disease duration in the regions of the metatarsus and heel, which were even more pronounced at M1-3. The high PTI for the metatarsal heads suggests that over time ulcerations, especially pressure-related ulcers, in these regions could potentially occur, as high PTIs have proved to be associated with ulcerations in diabetic patients [24]. Hence, our results may provide a more comprehensive understanding of this association. As a whole, it appears that the hallux can be used more as a significant reference parameter; the risk of plantar ulcers increase if peak pressure of hallux declines.
Multiple structural and functional factors have been documented to be linked with high plantar pressures; these include BMI, limited joint mobility, plantar soft tissue thickness, stiffness and callosities, metatarsal length, the presence of metatarsal deformities, and toe deformities [8]. However, we believe that the most important factor in our study was BMI. Concordant with the results reported by Birtane et al. [26] and Shen et al. [7], we found that BMI was positively correlated with total plantar pressure and that age was a contributor to plantar pressure. In a study by Barn et al., as one parameter of global features, age was demonstrated to be a weaker predictor than local effects (e.g., foot deformity) [11]. With respect to the role of VPT, patients with higher VPT had significantly increased PTI. These findings have been already reported by Shen et al. who proved that a VPT higher than $21 \mathrm{~V}$ resulted in a 2.33fold higher likelihood of abnormal plantar pressure [7].

This is a cross-sectional trial that represents our first step in investigating the influence of the duration of diabetes on plantar pressure. Our results substantiate those reported by others who have speculated on the magnitude of diabetes duration and demonstrated that longer DM duration is tied to increased VPT and decreased ABI $[7,27]$. Patients with active foot ulcer and deformity were excluded from our study population, which allowed our results to reflect the mode of plantar pressure distribution among diabetes patients in general. The final analysis was not affected by the gaping difference between the left and right plantar as a result of diabetic foot ulcers. Thus, this study overcomes the disadvantages of the sample selection seen in other studies. In a cross-sectional study, Barn et al. recruited only 163 participants to investigate the possible contributing factors from among the multiple parameters that can influence plantar pressure in high-risk diabetes patients, implying that diabetes duration is a weak indicator [11]. In another case study carried out in India, only 33 subjects with ages ranging from 40 to 75 years were divided into three groups to distinguish diabetic neuropathy groups, and the control subjects was defined as non-diabetic ones [12]. 
In contrast, we enrolled 1196 individuals in our study, and those with IGT were distinguished from those without diabetes in order to better substantiate the impact of diabetes duration. Consequently, our data may carry more weight in terms of representing the challenges of plantar pressure distribution in diabetes.

Several limitations to this study have to be considered to help understand the generalizability of our results. This study was a crosssectional analysis of patients with T2DM. Additional prospective studies are further required to determine the role of diabetes duration as an indicator of the alterations in plantar pressure. The current results may not reflect the relationship between diabetes duration and distribution of plantar pressure in younger Chinese individuals with diabetes due to the comparable higher mean ages of the diabetes population in our study, nearly 60 years or older. Moreover, it should be noted that the relatively poor health conditions observed in the non-diabetes individuals in our study was revealed as a higher incidence of complications and poor neural and vascular functions in the lower limbs. Therefore, larger and younger control groups are needed to confirm our findings.

\section{CONCLUSIONS}

To summarize, we found an upward trend in peak pressure on the hallux in patients with T2DM of $<5$ years duration, followed by a distinct downward slope with further progression of the disease. An uneven distribution of peak pressure was found at other locations, but the value was ultimately lower than that of the controls. No obvious trend was noted for the PTI; however, patients with T2DM of $>10$ years duration manifested a significantly sharp increase at the metatarsus and heel subregions. In addition, there was a fluctuation in the PTI of the hallux and MF, which was by large flat when compared with that of the controls. The reduced pressure of the hallux has been supposed to be transferred to the metatarsus; however, we observed that if peak pressure of the hallux declined, the risk of plantar ulcers increased. Stepwise multiple regression analysis demonstrated that BMI was the most distinct risk factor of abnormal peak pressure, while age, BMI, and VPT were predictors of a higher plantar PTI. Generally, our study shows the instructive significance of the measurement of plantar pressure and that the screening for plantar pressure as early as possible and adoption of offloading treatment could help to prevent the development of foot ulcers.

\section{ACKNOWLEDGEMENTS}

We thank the participants of the study. The authors are grateful to the participants of this study for their invaluable contributions to this work.

Funding. This research and the Rapid Service Fee was supported by grants from the National Science Foundation Items of China (81270397, 81770802 for Fang Liu), and Shanghai Municipal Education CommissionGaofeng Clinical Medicine (20152232 for Fang Liu).

Authorship. All named authors meet the International Committee of Medical Journal Editors (ICMJE) criteria for authorship for this article, take responsibility for the integrity of the work as a whole, and have given their approval for this version to be published.

Authorship Contributions. FL, JZ (third author), JZ (fourth author), JY, HC, YC, YB, and WJ designed the study. FL reviewed the manuscript. LX and HZ collected data. LX analyzed the data and drafted the manuscript.

Disclosures. Lei Xu, Hui Zeng, Jun Zhao, Jungong Zhao, Jun Yin, Hua Chen, Yimin Chai, Yuqian Bao, Fang Liu, and Weiping Jia have nothing to disclose.

Compliance with Ethics Guidelines. All procedures performed in studies involving human participants were in accordance with the Institutional Review Board and Ethics Committee of Shanghai Sixth people's Hospital 
affiliated to the Shanghai Jiao Tong University School of Medicine and with the 1964 Helsinki Declaration and its later amendments or comparable ethical standards. Informed consent was obtained from all individuals included in the study.

Data Availability. The datasets generated during and/or analyzed during the current study are available from the corresponding author on reasonable request.

Open Access. This article is distributed under the terms of the Creative Commons Attribution-NonCommercial 4.0 International License (http://creativecommons.org/licenses/ by-nc/4.0/), which permits any noncommercial use, distribution, and reproduction in any medium, provided you give appropriate credit to the original author(s) and the source, provide a link to the Creative Commons license, and indicate if changes were made.

\section{REFERENCES}

1. Akbari CM, Macsata R, Smith BM, Sidawy AN. Overview of the diabetic foot. Semin Vasc Surg. 2003;16(1):3-11.

2. Vinik A, Ullal J, Parson HK, Casellini CM. Diabetic neuropathies: clinical manifestations and current treatment options. Nat Clin Pract Endocrinol Metab. 2006;2(5):269-81.

3. Rathur HM, Boulton AJ. The neuropathic diabetic foot. Nat Clin Pract Endocrinol Metab. 2007;3(1):14-25.

4. Bus SA, Waaijman R. The value of reporting pressure-time integral data in addition to peak pressure data in studies on the diabetic foot: a systematic review. Clin Biomech (Bristol Avon). 2013;28(2):117-21.

5. Bus SA. Foot structure and footwear prescription in diabetes mellitus. Diabetes Metab Res Rev. 2008;24[Suppl 1]:S90-5.

6. Waaijman R, de Haart M, Arts ML, et al. Risk factors for plantar foot ulcer recurrence in neuropathic diabetic patients. Diabetes Care. 2014;37(6):1697-705.
7. Shen J, Liu F, Zeng $\mathrm{H}$, et al. Vibrating perception threshold and body mass index are associated with abnormal foot plantar pressure in type 2 diabetes outpatients. Diabetes Technol Ther. 2012;14(11):1053-9.

8. Guldemond NA, Leffers P, Walenkamp GH, et al. Prediction of peak pressure from clinical and radiological measurements in patients with diabetes. BMC Endocr Disord. 2008;8:16.

9. Song-Hua Y, Lu W, Kuan Z. Effects of different movement modes on plantar pressure distribution patterns in obese and non-obese Chinese children. Gait Posture. 2017;57:28-34.

10. Halawa MR, Eid YM, El-Hilaly RA, Abdelsalam MM, Amer AH. Relationship of planter pressure and glycemic control in type 2 diabetic patients with and without neuropathy. Diabetes Metab Syndr. 2018;12(2):99-104.

11. Barn R, Waaijman R, Nollet F, Woodburn J, Bus SA. Predictors of barefoot plantar pressure during walking in patients with diabetes, peripheral neuropathy and a history of ulceration. PLOS One. 2015;10(2):e0117443.

12. Periyasamy R, Anand S, Ammini AC. Prevalence of standing plantar pressure distribution variation in north Asian Indian patients with diabetes mellitus: a study to understand ulcer formation. Proc Inst Mech Eng H. 2013;227(2):181-9.

13. Tominaga M. Diagnostic criteria for diabetes mellitus. Rinsho Byori. 1999;47(10):901-8.

14. Kwon OY, Minor SD, Maluf KS, Mueller MJ. Comparison of muscle activity during walking in subjects with and without diabetic neuropathy. Gait Posture. 2003;18(1):105-13.

15. Maluf KS, Mueller MJ, Strube MJ, Engsberg JR, Johnson JE. Tendon Achilles lengthening for the treatment of neuropathic ulcers causes a temporary reduction in forefoot pressure associated with changes in plantar flexor power rather than ankle motion during gait. J Biomech. 2004;37(6):897906.

16. Bus SA, Maas M, de Lange A, Michels RP, Levi M. Elevated plantar pressures in neuropathic diabetic patients with claw/hammer toe deformity. J Biomech. 2005;38(9):1918-25.

17. Bacarin TA, Sacco ICN, Hennig EM. Plantar pressure distribution patterns during gait in diabetic neuropathy patients with a history of foot ulcers. Clinics (Sao Paulo). 2009;64(2):113-20. 
18. Lord M, Hosein R. A study of in-shoe plantar shear in patients with diabetic neuropathy. Clin Biomech (Bristol Avon). 2000;15(4):278-83.

19. Gooding GA, Stess RM, Graf PM, Moss KM, Louie KS, Grunfeld C. Sonography of the sole of the foot. Evidence for loss of foot pad thickness in diabetes and its relationship to ulceration of the foot. Invest Radiol. 1986;21(1):45-8.

20. Lavery LA, Peters EJG, Armstrong DG. What are the most effective interventions in preventing diabetic foot ulcers? Int Wound J. 2008;5(3):425-33.

21. Mueller MJ, Zou D, Bohnert KL, Tuttle LJ, Sinacore DR. Plantar stresses on the neuropathic foot during barefoot walking. Phys Ther. 2008;88(11):1375-84.

22. Abouaesha F, van Schie CH, Griffths GD, Young RJ, Boulton AJ. Plantar tissue thickness is related to peak plantar pressure in the high-risk diabetic foot. Diabetes Care. 2001;24(7):1270-4.
23. Masson EA, Hay EM, Stockley I, Veves A, Betts RP, Boulton AJ. Abnormal foot pressures alone may not cause ulceration. Diabet Med. 1989;6(5):426-8.

24. Hsi WL, Chai HM, Lai JS. Comparison of pressure and time parameters in evaluating diabetic footwear. Am J Phys Med Rehabil. 2002;81(11):822-9.

25. Veves A, Murray HJ, Young MJ, Boulton AJ. The risk of foot ulceration in diabetic patients with high foot pressure: a prospective study. Diabetologia. 1992;35(7):660-3.

26. Birtane $M$, Tuna $H$. The evaluation of plantar pressure distribution in obese and non-obese adults. Clin Biomech (Bristol Avon). 2004;19(10):1055-9.

27. Zhang J, Xue YM. The risk factors for abnormal ankle-brachial index in type 2 diabetic patients and clinical predictive value for diabetic foot. Zhonghua Nei Ke Za Zhi. 2013;52(11):951-5. 